\title{
Dependencia y adicción al smartphone de una muestra de jóvenes extremeños: diferencias por sexo y edad
}

\section{David Cerro Herrero}

Profesor en la Facultad de Formación del Profesorado de la Universidad de Extremadura davidcerro@unex.es

Jorge Rojo Ramos

Profesor en la Facultad de Educación de la Universidad de Extremadura

jorgerr@unex.es

\section{María de los Ángeles González González}

Doctoranda en la Facultad de Ciencias del Deporte de la Universidad de Extremadura magonzalezg@unex.es

\section{Miguel Madruga Vicente}

Profesor en la Facultad de Formación del Profesorado de la Universidad de Extremadura miguelmadruga@unex.es

Josué Prieto Prieto

Profesor doctor en la Escuela Universitaria de Educación y Turismo de la Universidad de Salamanca

\section{Extracto}

El uso del smartphone ha crecido en los últimos años, convirtiéndose en un elemento fundamental en la vida diaria de las personas; sin embargo, su utilización puede comportar algunos riesgos. Los jóvenes, población en constante evolución y aprendizaje, son más vulnerables a los riesgos de uso de los smartphones. El objetivo fue analizar la dependencia y adicción al smartphone en jóvenes extremeños. La muestra fue de 271 participantes en campamentos de verano de Extremadura en 2019, quienes contestaron al cuestionario elaborado para el estudio. Se observaron niveles elevados de dependencia y adicción al smartphone ( $51,3 \%$ de dependientes y $23,6 \%$ de adictos). También, se detectaron mayores niveles de adicción en los chicos que en las chicas (30,3\% versus $18,1 \%$ ) y en la edad de 16 a 18 años $(36 \%)$. Asimismo, el gasto económico en aplicaciones y juegos móviles es mayor en los hombres. Se evidencia así la problemática del uso y abuso del smartphone en los jóvenes. Podrían ser necesarios programas educativos para reducir los riesgos asociados al abuso de los smartphones.

Palabras clave: dependencia; adicción; smartphone; jóvenes; campamentos.

Fecha de entrada: 19-01-2020 / Fecha de revisión: 29-03-2020 / Fecha de aceptación: 30-03-2020

Cómo citar: Cerro Herrero, D., Rojo Ramos, J., González González, M. ${ }^{a}$ Á., Madruga Vicente, M. y Prieto Prieto, J. (2020). Dependencia y adicción al smartphone de una muestra de jóvenes extremeños: diferencias por sexo y edad. Tecnología, Ciencia y Educación, 17, 35-53. 


\title{
Smartphone dependence and addiction of a sample of young people from Extremadura: differences by sex and age
}

\author{
David Cerro Herrero \\ Jorge Rojo Ramos \\ María de los Ángeles González González \\ Miguel Madruga Vicente \\ Josué Prieto Prieto
}

\begin{abstract}
The use of the smartphone has grown in recent years, becoming a fundamental element in people's daily lives; however, its use may involve some risks. Young people, a population in constant evolution and learning, are more vulnerable to the risks of smartphone use. The aim was to analyse the dependence and addiction to smartphones in young people in Extremadura. The sample was 271 participants in summer camps in Extremadura in 2019, who answered the questionnaire developed for the study. High levels of dependence and addiction to the smartphone were observed ( $51.3 \%$ of dependents and $23.6 \%$ of addicts). Also, higher levels of addiction were detected in boys than in girls (30.3\% versus $18.1 \%)$ and in the age group $16-18$ years (36\%). Also, economic expenditure on mobile applications and games is higher in men. This shows the problem of smartphone use and abuse among young people. Educational programs may be needed to reduce the risks associated with smartphone abuse.
\end{abstract}

Keywords: dependence; addiction; smartphone; youth; camp.

Citation: Cerro Herrero, D., Rojo Ramos, J., González González, M. ${ }^{a}$ Á., Madruga Vicente, M. and Prieto Prieto, J. (2020). Smartphone dependence and addiction of a sample of young people from Extremadura: differences by sex and age. Tecnología, Ciencia y Educación, 17, 35-53. 


\section{Sumario}

1. Introducción

2. Método

2.1. Participantes

2.2. Instrumentos

2.3. Procedimiento

2.4. Análisis estadísticos

3. Resultados

4. Discusión

Referencias bibliográficas 


\section{Introducción}

En los últimos años, algunos estudios destacan que los smartphones se han instalado como elementos tecnológicos imprescindibles en la vida de los jóvenes (Ditrendia, 2019; Observatorio Nacional de las Telecomunicaciones y de la Sociedad de la Información [ONTSI], 2019). Sus prestaciones y funcionalidad han convertido este dispositivo en uno de los pilares fundamentales de los jóvenes en nuestra sociedad, ya que han supuesto un cambio en la manera de relacionarse entre los seres humanos y su entorno (González-Fernández, Salcines-Talledo y Ramírez-García, 2018).

Unido a este crecimiento tecnológico, internet nos ofrece el anonimato, la sincronía, la socialización y el sentirse miembro de una comunidad, así como la creación de una propia identidad digital (Beranuy y Sánchez-Carbonell, 2007). El smartphone, por su parte, ofrece la posibilidad de estar en permanente contacto con otras personas o realidades, posibilita los procesos de socialización, un aumento de la autonomía, la mejora en la gestión del tiempo y el acceso a la información. Así, este binomio internet-smartphone ha generado un gran atractivo para los jóvenes, lo que les ha llevado a formar parte de la denominada "generación Z» (nativos digitales) (Gaspar, 2016).

En la actualidad, la expansión del uso de internet en los países desarrollados adquiere una magnitud tan importante que resulta casi impensable no estar «conectado». Esta conectividad ha evolucionado, más allá de la necesidad operativa funcional (e-mail, GPS, mapas, etc.), hacia la necesidad de interaccionar con el entorno, principalmente a través de redes sociales (Morales, 2012). Según datos del Instituto Nacional de Estadística (INE) sobre equipamiento y uso de las tecnologías de la información y la comunicación (TIC) en los hogares en el año 2019, el 91,4 \% de los hogares tiene conexión a internet y un 91,2\% dispone de conexión de banda ancha. Asimismo, el 66 \% de los jóvenes con edades comprendidas entre los 10 y los 15 años dispone de móvil $(67,1 \%$ niñas y $65 \%$ niños) y el $92,9 \%$ utiliza internet. En personas de edades comprendidas entre los 16 y los 74 años, el uso de internet es del 90,7\%, y el $95 \%$ accede a la red a través del teléfono móvil (fuera de casa y del trabajo). Además, el 64,6 \% participa en redes sociales, siendo el porcentaje de féminas de un $67 \%$ y el masculino de un 62,1\% (INE, 2019). 
En España, las principales actividades que realiza la población general a través del móvil son aquellas relacionadas con la mensajería instantánea (85\%). El $97 \%$ de los jóvenes entre 14 y 24 años usa habitualmente las aplicaciones de mensajería WhatsApp, Telegram o Facebook Messenger para comunicarse, siendo lo más utilizado la interacción en grupos (un $65 \%$ de los niños entre 11 y 14 años participa en algún grupo) (Cánovas, 2014). Otras utilidades destacables son la visualización de vídeos online (82\%) y las relacionadas con el entretenimiento (49\%) (Ditrendia, 2019). Asimismo, se ha observado un incremento exponencial del número de descargas de aplicaciones móviles en los últimos años en nuestro país. Se estima que existen 23 millones de usuarios activos de aplicaciones móviles, los cuales tienen instaladas una media de unas 39 (Gaspar, 2016). Las aplicaciones más interesantes para los usuarios siguen siendo los juegos, seguidas de las relacionadas con la fotografía, las redes sociales y el entretenimiento. Los españoles dedican a las aplicaciones móviles el $60 \%$ del tiempo que pasan utilizando el teléfono móvil y tienen una percepción de uso muy distinta a la realidad, es decir, utilizan las aplicaciones mucho más de lo que realmente son conscientes. Desde el punto de vista del gasto económico, en 2018, en España, se descargaron un total de 1,2 millones de aplicaciones móviles y la población española gastó un total de 457,8 millones de euros en ellas (Ditrendia, 2019).

Como consecuencia de esta constante conexión a los teléfonos inteligentes o smartphones, se ha generado una necesidad hacia las aplicaciones móviles, hecho que ha podido desarrollar un sentimiento de dependencia, incluso patológica, en la vida de muchas personas y, sobre todo, en los adolescentes

Como consecuencia de esta constante conexión a los teléfonos inteligentes o smartphones, se ha generado una necesidad hacia este tipo de aplicaciones, hecho que ha podido desarrollar un sentimiento de dependencia, incluso patológica, en la vida de muchas personas y, sobre todo, en los adolescentes. Esta adicción, conocida como «nomofobia», puede generar conductas desmedidas e incluso compulsivas en el uso del smartphone (Gaspar, 2016). Si hablamos de «adicción», cabe señalar que, en 2018, el Ministerio de Sanidad, Consumo y Bienestar Social añadió la adicción a las nuevas tecnologías dentro de su Plan de Acción sobre Adicciones 2018-2020 y en la Estrategia Nacional sobre Adicciones 2017-2024 debido a los efectos negativos que produce en la población (Ministerio de Sanidad, Consumo y Bienestar Social, 2018a y 2018b).

Este tipo de adicción se engloba dentro de las denominadas «adicciones comportamentales» (Simó, Martínez, Ballester y Domínguez, 2017), es decir, no se refiere a que la persona deba consumir alguna sustancia (como habitualmente sucede con una adicción), sino que modifica los hábitos de la persona y puede tener influencia sobre su personalidad. 
Una de las principales variables afectadas de la personalidad es la autoestima. Se ha demostrado que personas con baja autoestima presentan un mayor riesgo de adicción a este tipo de dispositivos (Chi-Ying, 2020; Elhai, Dvorak, Levine y Hall, 2017).

Además, según Cánovas (2005) y Criado (2005), el uso inapropiado de los dispositivos también tiene efectos sobre otras esferas de la persona, como puede ser la de la economía personal, $y$, como consecuencia de ello, puede conllevar un gasto excesivo. Este gasto económico puede estar determinado por distintos factores, entre ellos el sexo. En este caso, se han desarrollado estudios en los que se muestra que las mujeres declaran tener más dificultades a la hora de controlar el gasto en aplicaciones y juegos para móvil (Chóliz, Villanueva y Chóliz, 2009; Ruiz-Olivares, Lucena, Pino y Herruzo, 2010); sin embargo, otros estudios mostraron que el gasto económico era mayor en los hombres (Aranda, Fuentes y García-Domingo, 2017).

Con todo ello, el presente estudio tiene como objetivo principal analizar la dependencia y adicción a los smartphones en una muestra de jóvenes extremeños, de edades comprendidas entre los 10 y los 18 años, participantes en la campaña de campamentos de verano de 2019, determinando si existen diferencias significativas en los niveles de dependencia y adicción en función del sexo y la edad. En línea de lo anteriormente expuesto, se formulan las siguientes hipótesis:

- H1. Los jóvenes extremeños de 10 a 18 años hacen un uso excesivo del smartphone y presentan altos niveles de dependencia de este.

- H2. Los niveles de dependencia del smartphone son similares en ambos sexos y más elevados en los grupos de mayor edad.

\section{Método}

\subsection{Participantes}

La muestra estuvo compuesta por 271 jóvenes extremeños que participaron en la campaña de campamentos de verano de 2019 desarrollada por el Instituto de la Juventud de la Junta de Extremadura. Fueron seleccionados mediante un método de muestreo no 
probabilístico, basado en un muestreo de conveniencia (Salkind, 1999) o muestra a propósito (Patton, 2002), debido a que todos los sujetos seleccionados eran participantes de los campamentos de verano que consintieron participar en el estudio.

Del total de participantes, un $55 \%$ (149) eran chicas y un $45 \%$ (122) chicos. Las edades estaban comprendidas entre los 10 y los 18 años. Se distribuyeron en un 18,1\% (49), entre 10 y 12 años; en un 63,5\% (172), entre 13 y 15 años; y en un 18,4\% (50), entre 16 y 18 años. Por nivel educativo, el 13,3\% (36) cursaba educación primaria; el 74,2\% (201), educación secundaria obligatoria; y el 12,5\% (34), bachillerato.

\subsection{Instrumentos}

Para la recogida de datos se diseñó un cuestionario ad hoc sobre uso del smartphone con cuatro preguntas que contenían datos de clasificación sociodemográficos (la edad, el sexo, la etapa educativa y el campamento).

Para evaluar el uso problemático de las aplicaciones asociadas a los smartphones se utilizó la escala de dependencia y adicción al smartphone (EDAS) (Aranda, Fuentes y GarcíaDomingo, 2017), constituida por 40 ítems en total, agrupados en tres componentes:

- Componente 1. «Uso, abuso y adicción al smartphone y sus aplicaciones». Compuesto por 30 ítems que evalúan el grado de dependencia al uso del móvil y sus aplicaciones de mensajería y redes sociales.

- Componente 2. «Rasgos de personalidad y autoestima». Compuesto por seis ítems que miden el nivel de introversión-extroversión y de autoncepto-autovalía de los participantes.

- Componente 3. «Gasto monetario en aplicaciones y juegos móviles». Constituido por cuatro ítems que recogen el gasto de dinero asociado a la compra de aplicaciones y juegos a través del smartphone.

El instrumento emplea una escala tipo Likert donde los valores de las respuestas oscilan de 1 (totalmente en desacuerdo) a 5 (totalmente de acuerdo).

Tras la inversión de los ítems indirectos, una puntuación más elevada implica mayor dependencia (en el componente 1); mayor autoestima, asociada a la imagen de los demás, y más grado de extroversión (en el componente 2); y mayor gasto (en el componente 3). Los puntos de corte ofrecidos por los percentiles 25 y 75 en cada uno de los componentes permiten obtener tres niveles (bajo, medio y alto) que facilitan la interpretación de los resultados. La fiabilidad del instrumento original presenta índices adecuados en jóvenes españoles para cada componente de $a^{1}=0,81, a^{2}=0,75$ y $a^{3}=0,71$, obtenidos por alfa de Cronbach. 


\subsection{Procedimiento}

El cuestionario fue elaborado y administrado en soporte digital utilizando la herramienta Google Forms, seleccionada a partir de criterios de funcionalidad y operatividad para los participantes, y a su practicidad, pues permite guardar las respuestas directamente en una hoja de cálculo y así facilitar la transformación de estas con la finalidad de realizar un análisis estadístico adecuado. La recogida de datos tuvo lugar entre los meses de julio y septiembre de 2019, durante el desarrollo de la campaña de campamentos de verano que desarrolla el Instituto de la Juventud de Extremadura.

Los participantes en el estudio recibieron información detallada de la finalidad del mismo durante los dos primeros días de actividad. Asimismo, fueron asistidos en grupos reducidos por un técnico experto de evaluación, quien ayudó a resolver las dudas existentes y a la comprensión de los ítems. Los participantes completaron el cuestionario, que les fue facilitado mediante enlace URL, de forma anónima y haciendo uso de sus smartphones personales, conectados a internet a través de la red wifi de la instalación. El tiempo necesario para responder al cuestionario fue de 10 minutos.

\subsection{Análisis estadísticos}

Las puntuaciones de los tres componentes fueron obtenidas mediante el valor medio de los ítems que agrupa cada uno de ellos, tras la inversión de los ítems indirectos que establece el instrumento. También se realizó un análisis de las correlaciones entre los diferentes componentes del instrumento mediante prueba Rho de Spearman para muestras no paramétricas.

Las pruebas t-student y chi-cuadrado de Pearson $\left(X^{2}\right)$ fueron utilizadas para analizar las diferencias entre las variables del estudio. Para analizar el efecto del sexo y la edad de los participantes, se realizó un ANOVA, tomando como variables dependientes los componentes de la escala y como factor, en cada caso, el sexo y la edad agrupada en tres niveles (10-12, 13-15 y 16-18). El análisis de los datos recogidos fue realizado con el Statical Package for Social Sciences (SPSS), versión 23.0 para Windows (IBM, Armonk, Nueva York).

\section{Resultados}

El cuadro 1 muestra los descriptivos de los componentes resultantes y del total de ítems de la escala utilizada, así como las puntuaciones medias por sexo y el nivel de significación estadística. 


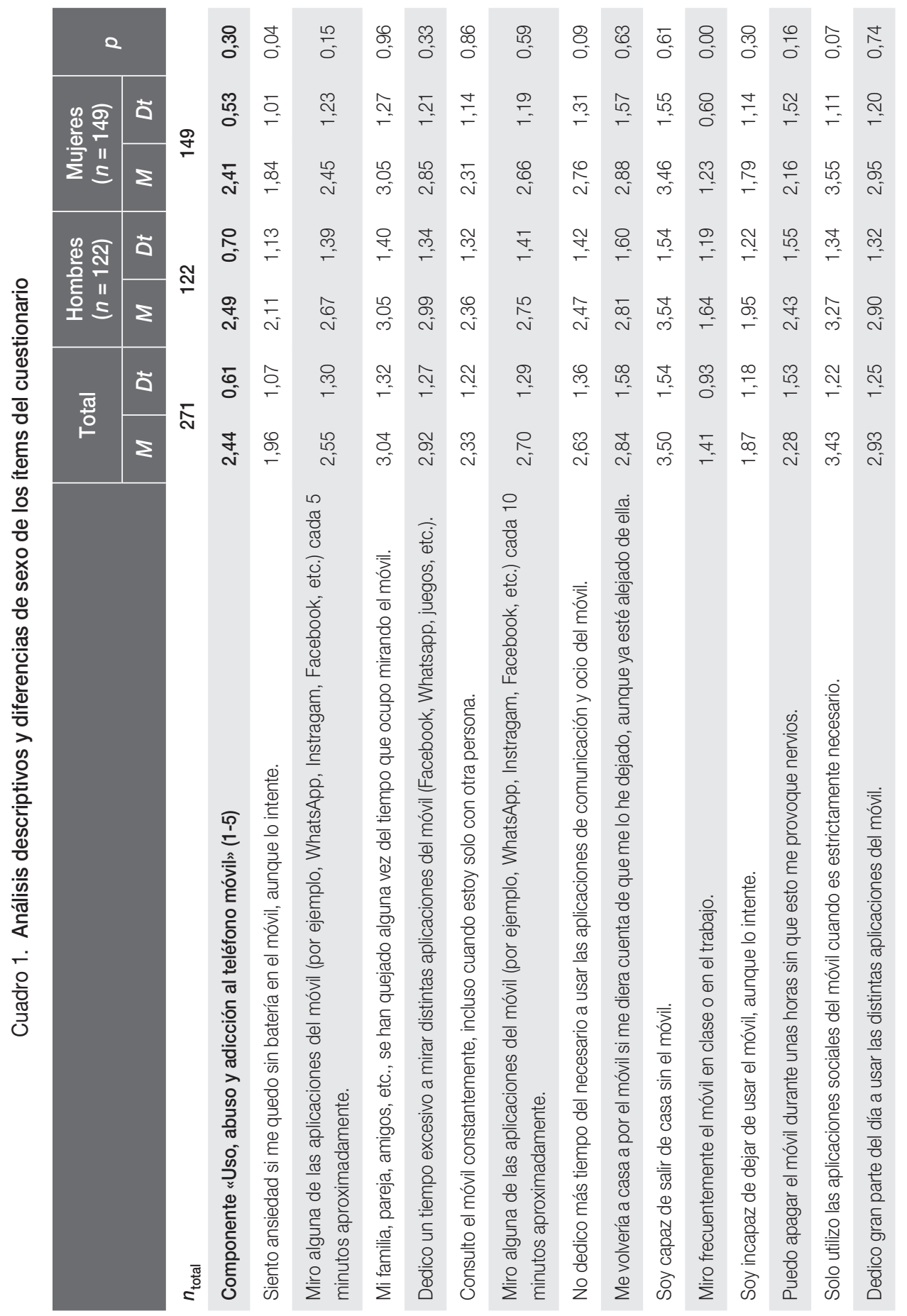




\begin{tabular}{|c|c|c|c|c|c|c|c|c|c|c|c|c|c|c|c|}
\hline \multicolumn{2}{|c|}{ e } & 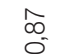 & ले & $\widetilde{\widetilde{m}}$ & 吕 & 8 & $\underset{\widetilde{N}}{\sigma}$ & $\begin{array}{l}\hat{2} \\
\hat{0}\end{array}$ & 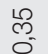 & ర్ & مొ & 8 & $\stackrel{\sim}{\sim}$ & $\stackrel{\infty}{m}$ & 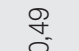 \\
\hline \multirow{2}{*}{ 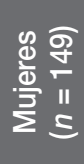 } & 苂 & $\stackrel{\text { m}}{\leftarrow}$ & $\underset{-}{\sigma}$ & $\stackrel{\sigma}{\check{L}}$ & $\stackrel{+}{\stackrel{0}{L}}$ & $\stackrel{ }{\stackrel{-}{=}}$ & $\underset{\sim}{\stackrel{g}{\leftarrow}}$ & 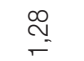 & $\underset{\sim}{\stackrel{ }{r}}$ & 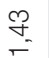 & $\underset{\simeq}{\simeq}$ & $\underset{\leftarrow}{\check{E}}$ & $\stackrel{\infty}{\leftarrow}$ & $\stackrel{\stackrel{2}{N}}{\sim}$ & $\underset{\sim}{\stackrel{\infty}{\sim}}$ \\
\hline & $\Sigma$ & $\begin{array}{l}\text { J } \\
\stackrel{\sim}{*}\end{array}$ & $\stackrel{+}{\stackrel{N}{r}}$ & $\begin{array}{l}\stackrel{2}{O} \\
\text { N }\end{array}$ & $\begin{array}{l}8 \\
\infty \\
\sim\end{array}$ & $\stackrel{\mathscr{\Omega}}{\leftarrow}$ & $\begin{array}{l}\text { L) } \\
\text { ণ }\end{array}$ & $\begin{array}{l}\bar{\sigma} \\
\stackrel{\sim}{*}\end{array}$ & $\underset{\sim}{\stackrel{\sim}{\sim}}$ & $\frac{\infty}{\stackrel{N}{c}}$ & $\overline{\text { i }}$ & $\frac{⿱ 亠}{\check{\sim}}$ & $\begin{array}{l}\text { Ő } \\
\text { N }\end{array}$ & $\begin{array}{l}\widetilde{\Xi} \\
\stackrel{\sim}{0}\end{array}$ & $\frac{N}{\bar{N}}$ \\
\hline \multirow{2}{*}{ 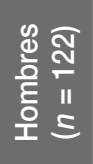 } & ప & 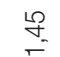 & $\stackrel{m}{=}$ & 우 & గ్ & m & $\hat{L}$ & $\hat{\omega}$ & $\stackrel{\infty}{\underset{N}{N}}$ & 足 & 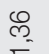 & ले & $\stackrel{\text { ? }}{\underset{N}{N}}$ & $\stackrel{\Im}{\sim}$ & $\stackrel{\text { D }}{\underline{N}}$ \\
\hline & $\Sigma$ & $\begin{array}{l}\otimes \\
\sim\end{array}$ & $\begin{array}{l}\infty \\
\stackrel{\infty}{-} \\
=-\end{array}$ & $\begin{array}{l}\bar{i} \\
\sim\end{array}$ & 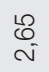 & \begin{tabular}{l}
$\approx$ \\
\multirow{\sim}{*}{}
\end{tabular} & $\begin{array}{l}\stackrel{2}{0} \\
\stackrel{\sim}{n}\end{array}$ & $\begin{array}{l}\infty \\
\stackrel{\infty}{\sim} \\
\sim\end{array}$ & $\begin{array}{l}\infty \\
\stackrel{\leftrightarrow}{\sim} \\
\sim\end{array}$ & $\begin{array}{l}\stackrel{\sim}{\sim} \\
\underset{N}{\sim}\end{array}$ & 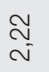 & $\begin{array}{l}\otimes \\
\sim\end{array}$ & $\frac{10}{\sim}$ & $\begin{array}{l}\hat{N} \\
\hat{N}\end{array}$ & $\begin{array}{l}\stackrel{\infty}{\sim} \\
\stackrel{\sim}{\sim}\end{array}$ \\
\hline \multirow{2}{*}{ त्ञँ } & 䓃 & $\stackrel{\infty}{m}$ & $\stackrel{\circ}{\sigma}$ & $\underset{\underset{N}{(}}{\stackrel{2}{2}}$ & م & $\stackrel{\text { શ }}{\sim}$ & న్ర & $\bar{m}$ & $\stackrel{\mathscr{N}}{\sim}$ & $\stackrel{\mathscr{Q}}{\mathscr{q}}$ & 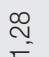 & 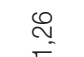 & $\approx$ & $\widetilde{ల ్}$ & $\stackrel{\infty}{\sim}$ \\
\hline & $\Sigma$ & $\begin{array}{l}\otimes \\
\stackrel{\sim}{*}\end{array}$ & $\stackrel{\infty}{-}$ & $\frac{m}{c}$ & $\underset{\sim}{\stackrel{N}{N}}$ & ì & $\begin{array}{l}\infty \\
\infty \\
\sim\end{array}$ & $\begin{array}{c}\text { ల) } \\
\text { N }\end{array}$ & $\stackrel{\text { g }}{\sim}$ & 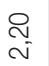 & $\frac{0}{\stackrel{\sim}{\sim}}$ & $\begin{array}{l}\infty \\
\stackrel{\infty}{\sim} \\
\sim\end{array}$ & $\underset{\sim}{\stackrel{\sim}{~}}$ & $\begin{array}{l}\stackrel{8}{0} \\
\text { N }\end{array}$ & $\underset{\underset{\sim}{\approx}}{\stackrel{\sim}{\sim}}$ \\
\hline & & 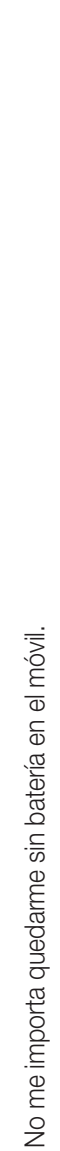 & 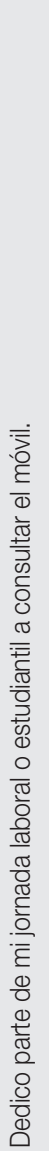 & 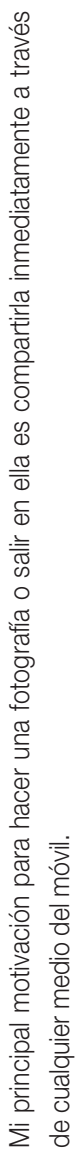 & 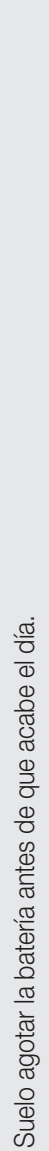 & 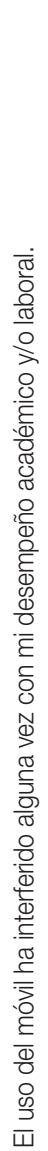 & 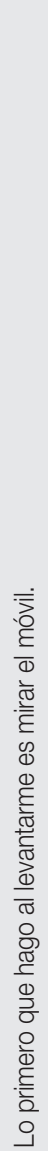 & 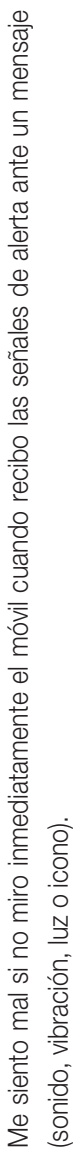 & 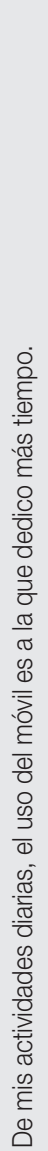 & 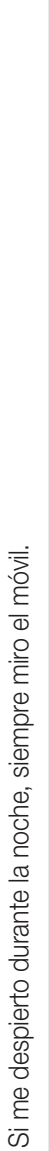 & 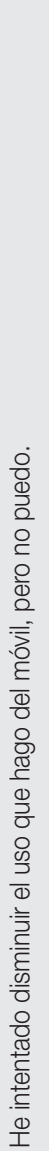 & 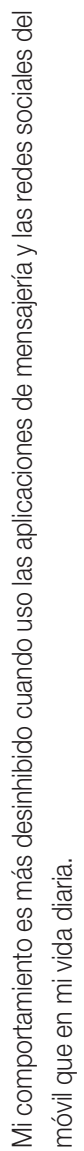 & 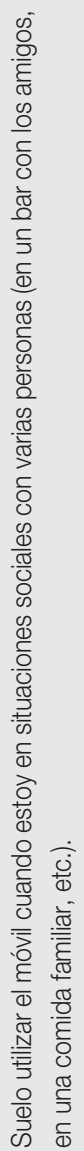 & 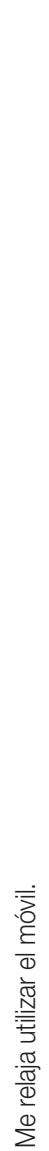 & 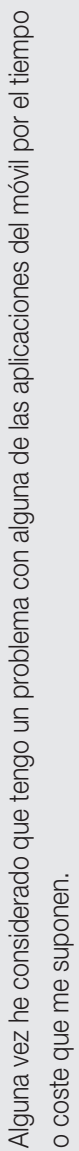 \\
\hline
\end{tabular}




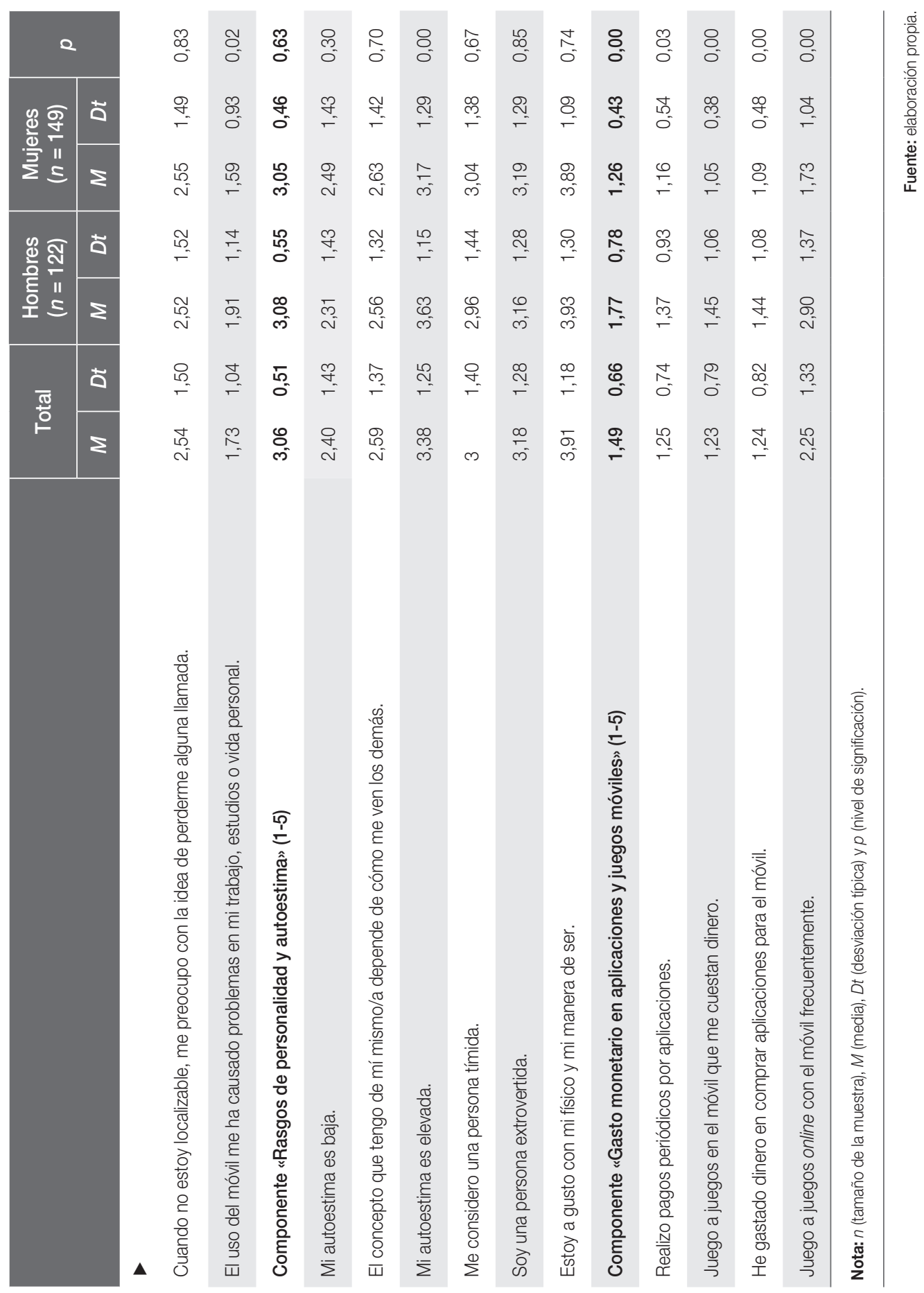


En relación al primer componente, «Uso, abuso y adicción al smartphone y sus aplicaciones», los participantes en el estudio muestran una puntuación media de 2,44 (Dt=0,61). La media de los hombres fue mayor que la mostrada por las mujeres: $M_{\text {hombres }}=2,49$ $(D t=0,70)$ y $M_{\text {mujeres }}=2,41(D t=0,53)$. En relación a los ítems que agrupa este componente, las puntuaciones más elevadas (mayor dependencia), tanto en hombres como en mujeres, fueron obtenidas en la incapacidad para salir de casa sin el móvil $(M=3,50$; $D t=1,54)$ y en lo referido al uso de aplicaciones sociales cuando no es necesario $(M=$ $=3,43 ; D t=1,22$ ). Se obtuvieron diferencias significativas en las medias de las puntuaciones respecto al sexo, concretamente en los ítems referidos a un comportamiento más desinhibido en el uso de aplicaciones de mensajería y redes sociales $(t=-3,34 ; p=0,00)$, con puntuaciones $M_{\text {hombres }}=2,66(D t=1,34)$ y $M_{\text {mujeres }}=2,14(D t=1,14)$, y en el "uso frecuente del móvil en clase» $(t=-3,41 ; p=0,00)$, con puntuaciones $M_{\text {hombres }}=1,64(D t=$ $=1,19)$ y $M_{\text {mujeres }}=1,23(D t=0,60)$.

La puntuación media del segundo componente, «Rasgos de personalidad y autoestima», fue de 3,06 $(D t=0,51): M_{\text {hombres }}=3,08(D t=0,55)$ y $M_{\text {mujeres }}=3,05(D t=0,46)$. Las puntuaciones más elevadas en los ítems que agrupa el componente fueron en «Estoy a gusto con mi físico y mi manera de ser» $(M=3,91 ; D t=1,18)$ y en «Mi autoestima es elevada» $(M=3,38, D t=1,25)$.

En el tercer componente, referido al «Gasto monetario en aplicaciones y juegos móviles», la puntuación media fue de $1,49(D t=0,66): M_{\text {hombres }}=1,77(D t=0,78)$ y $M_{\text {mujeres }}=1,26$ $(D t=0,43)$, obteniendo diferencias significativas en la comparación de medias entre el sexo $(t=-6,47 ; p=0,00)$.

El efecto de la edad sobre las respuestas no fue significativo en ninguno de los componentes tras realizar el análisis de ANOVA de un factor.

Igualmente, el análisis post hoc, efectuado con la prueba Bonferroni, tras asumir la homogeneidad de varianzas, no mostró diferencias entre los grupos de edad establecidos.

En el cuadro 2 podemos observar las frecuencias de hombres y mujeres en los tres niveles asociados a las puntuaciones en cada uno de los componentes de la escala, establecidos por los puntos de corte ofrecidos por los percentiles 25 y 75 (componente 1: $P 25=2,00$ y $P 75=2,87$; componente $2: P 25=2,66$ y $P 75=3,34$; componente $3: P 25=$ $=1,00$ y $P 75=1,75)$.

Se encontraron diferencias en la proporción de hombres y mujeres en el gasto monetario en aplicaciones y juegos móviles, con mayor proporción de hombres que reportan nivel de gasto alto $(32,8 \%)$ que de mujeres $(4,7 \%)$, confirmando una asociación entre el gasto monetario y el sexo $\left(X^{2}=5,92\right.$; coeficiente de contingencia $\left.=0,42 ; p=0,00\right)$. 
Cuadro 2. Distribución de frecuencias en los niveles de los componentes de la EDAS según sexo

\begin{tabular}{|c|c|c|c|c|c|c|}
\multirow{2}{*}{ Sexo } & \multicolumn{2}{|c|}{ Hombres (122) } & \multicolumn{2}{|c|}{ Mujeres (149) } & \multicolumn{2}{|c|}{ Total (271) } \\
\cline { 2 - 8 } & $n$ & $\%$ & $n$ & $\%$ & $n$ & $\%$ \\
\hline
\end{tabular}

Uso, abuso y adicción al smartphone

\begin{tabular}{lrrrrrr}
\hline Sin dependencia & 30 & 24,60 & 38 & 25,50 & 68 & 25,10 \\
Dependencia & 55 & 45,10 & 84 & 56,40 & 139 & 51,30 \\
Adicción & 37 & 30,30 & 27 & 18,10 & 64 & 23,60 \\
\hline Rasgos de personalidad y autoestima & & & & & & \\
\hline Bajo & 19 & 15,60 & 23 & 15,40 & 42 & 15,50 \\
Medio & 76 & 62,30 & 102 & 68,50 & 178 & 65,70 \\
Alto & 27 & 22,10 & 24 & 16,10 & 51 & 18,80 \\
\hline
\end{tabular}

Gasto monetario en aplicaciones y juegos móviles

\begin{tabular}{lllllll}
\hline Gasto bajo & 21 & 17,20 & 81 & 54,40 & 102 & 37,60 \\
Gasto medio & 61 & 50 & 61 & 40,90 & 122 & 45 \\
Gasto alto & 40 & 32,80 & 7 & 4,70 & 47 & 17,30 \\
\hline
\end{tabular}

En relación con la edad, los resultados muestran una asociación entre el componente de rasgos de personalidad y la edad $\left(X^{2}=14,91\right.$; coeficiente de contingencia $\left.=0,23 ; p=0,05\right)$. Se encontraron diferencias en las proporciones de grupos de edad establecidos, siendo los jóvenes de 13 a 15 años los que representan una mayor proporción $(22,1 \%)$ respecto a los otros dos grupos de edad, de un mayor riesgo en rasgos de personalidad y autoestima en relación con el uso de los smartphones (véase cuadro 3).

Cuadro 3. Distribución de frecuencias en los niveles de los componentes de la EDAS según edad

\begin{tabular}{|l|c|c|c|c|c|c|c|c|}
\multirow{2}{*}{ Edad } & \multicolumn{2}{|c|}{$10-12(49)$} & \multicolumn{1}{|c|}{$13-15(172)$} & \multicolumn{2}{|c|}{$16-18(50)$} & \multicolumn{2}{c|}{ Total (271) } \\
\cline { 2 - 8 } & $n$ & $\%$ & $n$ & $\%$ & $n$ & $\%$ & $n$ & $\%$ \\
\hline
\end{tabular}

Uso, abuso y adicción al smartphone

\begin{tabular}{lrlllllll}
\hline Sin dependencia & 13 & 26,50 & 49 & 28,50 & 6 & 12 & 68 & 25,10 \\
Dependencia & 25 & 51 & 88 & 51,20 & 26 & 52 & 139 & 51,30 \\
\hline Adicción & 11 & 22,40 & 35 & 20,30 & 18 & 36 & 64 & 23,60 \\
\hline
\end{tabular}




\begin{tabular}{|c|c|c|c|c|c|c|c|c|}
\hline \multirow{2}{*}{ Edad } & \multicolumn{2}{|c|}{$10-12(49)$} & \multicolumn{2}{|c|}{$13-15(172)$} & \multicolumn{2}{|c|}{$16-18(50)$} & \multicolumn{2}{|c|}{ Total (271) } \\
\hline & $n$ & $\%$ & $n$ & $\%$ & $n$ & $\%$ & $n$ & $\%$ \\
\hline \multicolumn{9}{|c|}{ 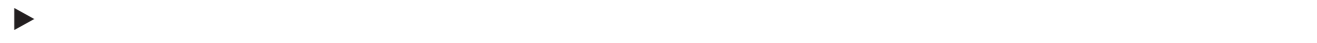 } \\
\hline \multicolumn{9}{|c|}{ Rasgos de personalidad y autoestima } \\
\hline Bajo & 8 & 16,30 & 33 & 19,20 & 1 & 2 & 42 & 15,50 \\
\hline Medio & 37 & 75,50 & 101 & 58,70 & 40 & 80 & 178 & 65,70 \\
\hline Alto & 4 & 8,20 & 38 & 22,10 & 9 & 18 & 51 & 18,80 \\
\hline \multicolumn{9}{|c|}{ Uso, abuso y adicción al smartphone } \\
\hline Gasto bajo & 14 & 28,60 & 73 & 42,40 & 15 & 30 & 102 & 37,60 \\
\hline Gasto medio & 25 & 51 & 72 & 41,90 & 25 & 50 & 122 & 45 \\
\hline Gasto alto & 10 & 20,40 & 27 & 15,70 & 10 & 20 & 47 & 17,30 \\
\hline
\end{tabular}

El cuadro 4 muestra la matriz de correlaciones de los componentes y de los niveles de la EDAS con las variables de sexo y grupos de edad. El sexo mostró un grado de correlación fuerte, a nivel de significatividad de estadístico menor de 0,01, con el tercer componente de gasto monetario en aplicaciones y juegos $(r=0,38 ; p=0,00)$ e, igualmente, en relación con los niveles establecidos en este $(r=0,45 ; p=0,00)$.

En relación con los grupos de edad, no mostró correlación significativa con alguno de los componentes. En el caso de las correlaciones con los niveles de los componentes de la EDAS, mostró relaciones moderadas, a nivel de significatividad de estadístico menor de 0,05, con el primer componente de uso, abuso y adicción $(r=0,12 ; p=0,04)$ y con el segundo componente de rasgos de personalidad $(r=0,13 ; p=0,04)$.

Cuadro 4. Correlaciones entre los componentes y los niveles de la EDAS y las variables de sexo y grupos de edad

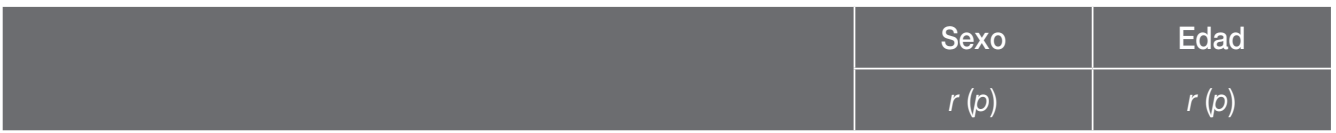

Componentes

Uso, abuso y adicción al smartphone.

$0,07(0,28) \quad 0,08(0,19)$

Rasgos de personalidad y autoestima.

$0,03(0,62)$

$0,12(0,06)$

Gasto monetario en aplicaciones y juegos móviles.

$0,38(0,00)^{\star \star} \quad-0,07(0,27)$ 


\begin{tabular}{|c|c|c|}
\hline & Sexo & Edad \\
\hline & $r(p)$ & $r(p)$ \\
\hline \multicolumn{3}{|l|}{$\triangleright$} \\
\hline \multicolumn{3}{|l|}{ Niveles } \\
\hline Uso, abuso y adicción al smartphone. & $0,09(0,12)$ & $0,12(0,04)^{\star}$ \\
\hline Rasgos de personalidad y autoestima. & $0,05(0,41)$ & $0,13(0,04)^{*}$ \\
\hline Gasto monetario en aplicaciones y juegos móviles. & $0,45(0,00)^{\star *}$ & $-0,01(0,91)$ \\
\hline
\end{tabular}

Nota: Rho de Spearman (r); la correlación es significativa al nivel ${ }^{* *} p<0,01 ;{ }^{*} p<0,05$.

Fuente: elaboración propia.

\section{Discusión}

Los resultados del presente estudio muestran la relación de dependencia y el abuso hacia el uso del smartphone en una muestra de jóvenes extremeños; resultados que son consistentes con los obtenidos en estudios previos a nivel nacional y mundial (Chóliz et al., 2009; Lu-Ying, 2019; Oliva et al., 2012). Si bien un estudio previo reportó puntuaciones muy elevadas acerca del uso diario de dispositivos móviles y del acceso a internet en adolescentes en Extremadura (Pulido, 2015), los niveles de dependencia y adicción al smartphone no habían sido comprobados en la región extremeña hasta ahora.

En relación con el uso, el abuso y la adicción en los jóvenes estudiados, podemos decir que, de cada cuatro jóvenes, tanto en hombres como en mujeres, tan solo uno de ellos no tiene dependencia $(25,1 \%)$, y el resto es clasificado como dependiente o adicto al uso del smartphone. Estos valores son ligeramente más bajos que los reportados en el estudio de validación del instrumento (Aranda et al., 2017), utilizado con una muestra de mayor edad (media de 22,38 años y rango entre 18-40 años).

Los hallazgos del presente estudio son consistentes con la literatura previa, que evidencia una mayor disposición a la dependencia y adicción en el género masculino (Beranuy, Chamarro, Graner y Carbonell, 2009; Lu-Ying, 2019; Oliva et al., 2012). Concretamente, en este estudio, las diferencias de sexo fueron halladas especialmente en relación con el mayor gasto monetario en aplicaciones y juegos móviles de los hombres. En este sentido, Valdez y Rufino (2020) observaron tendencias similares en un reciente estudio en jóvenes universitarios.

A pesar de que los resultados no muestran diferencias significativas en relación con los grupos de edad establecidos, en la línea de estudios previos (Cruces-Montes, Guil-Bozal, Sánchez-Torres y Pereira-Núñez, 2016; De la Villa-Moral y Suárez, 2016), se observa una tendencia positiva entre la edad y el nivel de dependencia y adicción al móvil, con mayor porcentaje de jóvenes adictos en el grupo de edad 16-18 años. No obstante, parece que 
el uso abusivo del móvil e internet se normaliza con la edad hacia un uso más profesional, menos lúdico y con no tantos efectos negativos (Beranuy et al., 2009).

El gasto económico puede ser un criterio determinante a la hora de establecer si hay dependencia o no (Griffiths, 1995), además de comprometer la economía familiar por el pago de la factura. Adentrarse a edades tempranas en los juegos de pago online a través del smartphone puede ser considerado como un factor de riesgo en el inicio al juego patológico sensiblemente «masculinizado», que tiene asociada consecuencias psicosociales negativas (Ministerio de Sanidad, Consumo y Bienestar Social, 2018a y 2018b).

La autoestima ha sido uno de los rasgos más estudiados en relación con el abuso del móvil, de modo que una baja autoestima puede llevar a un uso inapropiado del móvil, o bien un uso inapropiado puede generar consecuencias negativas que impacten en la autoestima (Ha, Petersen y Sharp, 2008). Los resultados del presente estudio no hallaron relaciones significativas en el componente de rasgos de personalidad y autoestima con relación al sexo. Sin embargo, sí se encontraron diferencias significativas de este componente con relación a la edad, siendo mayor en el grupo de 13-15 años. En este sentido, estudios previos han situado tanto la baja autoestima como el rasgo de personalidad más consistentemente asociado a la adicción, siendo las mujeres el grupo más vulnerable, de modo que los sujetos con baja autoestima manifiestan mayor tendencia a ser adictos (Cuesta, 2019) y aumentan las probabilidades de puntuar más alto en conflictos interpersonales asociados al uso problemático de internet (De la Villa y Fernández Domínguez, 2019).

La dependencia al teléfono móvil en adolescentes se ha relacionado con trastornos psicosociales, como ansiedad, depresión, o trastornos del sueño (Demirci, Akgönül y Akpinar, 2015; Gao, Li y Zhu, 2016; Thomée, Härenstam y Hagberg, 2011). Los adolescentes son considerados un segmento poblacional emocionalmente frágil (Augner y Hacker, 2012) que justifica la necesidad de promover programas psicoeducativos para fomentar un uso responsable del móvil.

Así, el entorno escolar y las familias han sido siempre situados como agentes principales en la prevención e intervención de estos programas (Tortosa y Montañes, 2017), pero existen otros contextos apropiados para estos, como son los relacionados con las actividades de ocio y deportivas, que pueden reducir los niveles de dependencia al smartphone (Liu, Xiao, Yang y Loprinzi, 2019).

El presente estudio contiene algunas limitaciones que deben ser consideradas. Una de ellas es el método de selección de la muestra, ya que esta no se ha realizado de acuerdo con procedimientos aleatorios, debido a que la muestra estaba limitada a jóvenes extremeños participantes en la campaña de campamentos descrita previamente. De igual modo, convenimos que una muestra más amplia podría contribuir a la obtención de resultados más representativos y con ello se conseguiría una mejora en la validez externa. Asimismo, la participación de jóvenes procedentes de diferentes comunidades autónomas permitiría obtener resultados que reflejasen la realidad de otros jóvenes en relación con las variables estudiadas. Por otro lado, la recogida de datos mediante autoinforme puede estar en cierto 
modo condicionada por la deseabilidad social. Finalmente, el estudio correlacional limita extraer conclusiones de causalidad entre las variables estudiadas.

Como línea de investigación futura se plantea afrontar nuevos estudios que, además de profundizar en el análisis de la situación actual, planteen intervenciones para educar en el uso responsable de estos dispositivos y para reducir los aspectos negativos de abuso, dependencia y adicción a través de diversas herramientas educativas o formativas. En este sentido, se plantea analizar si los campamentos de verano podrían considerarse como lugares para la «desintoxicación digital», basados en la participación en actividades de ocio y deportivas, alejadas de las nuevas tecnologías, y donde hubiera largos periodos de tiempo con restricción en el uso del móvil.

\section{Referencias bibliográficas}

Aranda, M. ${ }^{a}$, Fuentes, V. y García-Domingo, M. (2017). «No sin mi smartphone»: elaboración y validación de la escala de dependencia y adicción al smartphone (EDAS). Terapia Psicológica, 35(1), 35-45. doi: http:// dx.doi.org/10.4067/S0718-480820170001 00004.

Augner, C. y Hacker, G. W. (2012). Association between problematic mobile phone use and psychological parameters in young adults. International Journal of Public Health, 57(2), 437-441. doi: https://doi.org/10.10 07/s00038-011-0234-z.

Beranuy, M. y Sánchez-Carbonell, X. (2007). El móvil en la sociedad de la comunicación. La movilización de la sociedad. En A. Talarn (Coord.), Globalización y salud mental (pp. 369-392). Barcelona, España: Herder.

Beranuy, M., Chamarro, A., Graner, C. y Carbonell, X. (2009). Validación de dos escalas breves para evaluar la adicción a internet y el abuso de móvil. Psicothema, 21(3), 480-485. Recuperado de <http://hdl. handle.net/2072/205481 > (consultado el 12 de junio de 2019).
Cánovas, G. (2005). Seguridad infantil y costumbres de los menores en el empleo de la telefonía móvil. Madrid, España: Protégeles. Recuperado de <http://www.tecnoeduca.net/ sites/default/files/archivos/estudio_telefonia_ protegeles.pdf> (consultado el 7 de mayo de 2019).

Cánovas, G. (2014). Menores de edad y conectividad móvil en España: tablets y smartphones. Madrid, España: Protégeles. Recuperado de <https://kidsandteensonline. files.wordpress.com/2014/07/estudio_mo vil_smartphones_tablets_protegeles.pdf> (consultado el 7 de mayo de 2019).

Castillo, M. ${ }^{a}$ y Ruiz, R. (2019). La percepción de riesgo y su relación con el uso problemático del teléfono móvil en adolescentes. Reis: Revista Española de Investigaciones Sociológicas, 168, 21-34. doi: http://dx.doi. org/10.5477/cis/reis.168.21.

Chi-Ying, C. (2020). Smartphone addiction: psychological and social factors predict the use and abuse of a social mobile application. Information, Communication \& Society, 23(3), 454-467. doi:10.1080/1369118X.2018. 1518469. 
Chóliz, M., Villanueva, V. y Chóliz, M. C. (2009). Ellas, ellos y su móvil: uso, abuso (¿y dependencia?) del teléfono móvil en la adolescencia. Revista Española de Drogodependencias, 34(1), 74-88. Recuperado de <http://hdl.handle.net/10550/22402> (consultado el 7 de mayo de 2019).

Criado, M. Á. (2005). Enfermos del móvil. Ariadn@, 218. Recuperado de <http://www. el-mundo.es/ariadna/2005/218/11063 27395.html> (consultado el 7 de mayo de 2019).

Cruces-Montes, S. J., Guil-Bozal, R., SánchezTorres, N. y Pereira-Núñez, J. A. (2016). Consumo de nuevas tecnologías y factores de personalidad en estudiantes universitarios. Revista de Comunicación y Ciudadanía Digital, 5(2), 203-228. doi: https://doi. org/10.25267/COMMONS.2016.v5.i2.09.

Cuesta, V. (2019). Bases psicológicas de la adicción al «smartphone» (Tesis doctoral). Universidad Complutense de Madrid. Recuperado de <https://eprints.ucm.es/54208/ 1/T40984.pdf> (consultado el 9 de enero de 2020).

Demirci, K., Akgönül, M. y Akpinar, A. (2015). Relationship of smartphone use severity with sleep quality, depression, and anxiety in university students. Journal of Behavioral Addictions, 4(2), 85-92. doi: https://doi. org/10.1556/2006.4.2015.010.

Ditrendia (2019). Informe mobile en España y en el mundo 2019. Recuperado de <https:// mktefa.ditrendia.es/informe-mobile-2019> (consultado el 9 de enero de 2020).

Elhai, J. D., Dvorak, R. D., Levine, J. C. y Hall, B. J. (2017). Problematic smartphone use: a conceptual overview and systematic review of relations with anxiety and depression psychopathology. Journal of Affective Disorders, 207, 251-259. doi: 10.1016/j.jad. 2016.08.030.

Gao, Y., Li, A., Zhu, T., Liu, X. y Liu, X. (2016). How smartphone usage correlates with social anxiety and loneliness. PeerJ, 4: e2197. doi: https://doi.org/10.7717/peerj.2197.
Gaspar, S. (2016). Bases psicosociales del uso del smartphone en jóvenes: un análisis motivacional y cross-cultural (Tesis doctoral). Universidad Complutense de Madrid. Recuperado de <https://eprints.ucm.es/35447/1/T36788. pdfs (consultado el 12 de junio de 2019).

González-Fernández, N., Salcines-Talledo, I. y Ramírez-García, A. (2018). Dispositivos móviles -smartphones \& tablets- y comunicación en familia. Diseño de un focus group. Prisma Social: Revista de Investigación Social, 20, 21-39. Recuperado de $<$ https://dialnet.unirioja.es/descarga/articu lo/6360022.pdf> (consultado el 7 de mayo de 2019).

Griffiths, M. D. (1995). Technological addictions. Clinical Psychology Forum, 76, 14-19.

Ha, C., Petersen, N. y Sharp, C. (2008). Narcissism, self-esteem, and conduct problems. European Child \& Adolescent Psychiatry, 17(7), 406-413. doi: https://doi.org/10.1007/ s00787-008-0682-z.

INE. (2019). Encuesta sobre equipamiento y uso de TIC en los hogares. Recuperado de $<$ https://www.ine.es/dyngs/INEbase/es/operacion.htm?c=Estadistica_C\&cid=12547 6176741 \&menu=ultiDatos\&idp $=1254735976$ 608> (consultado el 9 de enero de 2020).

Liu, S., Xiao, T., Yang, L. y Loprinzi, P. D. (2019). Exercise as an alternative approach for treating smartphone addiction: a systematic review and meta-analysis of random controlled trials. International Journal of Environmental Research and Public Health, 16(20), 1-16. doi: https://doi.org/10.3390/ ijerph16203912.

Lu-Ying, N. I. U. (2019). The mediating role of self-differentiation in the relationship between college students' cell phone dependence and family function. In 4th Annual International Conference on Social Science and Contemporary Humanity Development (SSCHD 2018). Atlantis Press. doi: https:// doi.org/10.2991/sschd-18.2019.2. 
Ministerio de Sanidad, Consumo y Bienestar Social. (2018a). Estrategia nacional sobre adicciones 2017-2024. Madrid: Secretaría General Técnica. Centro de Publicaciones. Delegación del Gobierno para el Plan Nacional sobre Drogas.

Ministerio de Sanidad, Consumo y Bienestar Social. (2018b). Plan de acción sobre adicciones 2018-2020. Madrid: Secretaría General Técnica. Centro de Publicaciones. Delegación del Gobierno para el Plan Nacional sobre Drogas.

Morales, E. (2012). El smartphone como motor de nueva incertidumbre social. Prisma Social: Revista de Investigación Social, 8, 87-115. Recuperado de <https://dialnet.uni rioja.es/descarga/articulo/4004217.pdf> (consultado el 7 de mayo de 2019).

Oliva, A., Hidalgo, M. ${ }^{a}$ V., Moreno, C., Jiménez, L., Jiménez, A., Antolín, L. y Ramos, P. (2012). Uso y riesgo de adicciones a las nuevas tecnologías entre adolescentes y jóvenes andaluces. Sevilla: Aguaclara. Recuperado de <https://idus.us.es/xmlui/bits tream/handle/11441/67723/uso_riesgo. pdf?sequen> (consultado el 7 de mayo de 2019).

ONTSI. (2019). La sociedad en red. Transformación digital en España. Informe anual 2018. Madrid: ONTSI. doi: http://doi.org/10. 30923/1989-7424-2019.

Patton, M. Q. (2002). Qualitative Research and Evaluation Methods. Thousand Oaks, California: Sage.

Pulido, J. P. (2015). Estudio sobre el uso de dispositivos móviles en niños y adolescentes en Extremadura. Gobierno de Extremadura. Consejería de Educación y Cultura.

Ruiz-Olivares, R., Lucena, V., Pino, M. ${ }^{a}$ J. y Herruzo, J. (2010). Análisis de comportamientos relacionados con el uso/abuso de internet, teléfono móvil, compras y juego de estudiantes universitarios. Adicciones, 22, 301-310. doi: http://hdl.handle.net/10 396/11554.
Salkind, N. J. (1999). Exploring Research. Englewood Cliffs, Nueva Jersey, EE. UU.: Prentice Hall.

Simó, C., Martínez, A., Ballester, M. ${ }^{a}$ L. y Domínguez, A. (2017). Instrumentos de evaluación del uso problemático del teléfono móvil/smartphone. Health and Addictions, 17(1), 5-14. Recuperado de <https://www. redalyc.org/pdf/839/83949782001.pdf> (consultado el 12 de junio de 2019).

Thomée, S., Härenstam, A. y Hagberg, M. (2011). Mobile phone use and stress, sleep disturbances, and symptoms of depression among young adults: a prospective cohort study. BMC Public Health, 11, 66-72. doi: https://doi.org/10.1186/1471-2458-11-66.

Tortosa, E. M. y Montañés, M. C. (2017). Eficacia del programa de prevención PrevTec 3.1. Un estudio de uso de móviles en adolescentes (Tesis doctoral). Universitat de València. Recuperado de <http://roderic.uv. es/bitstream/handle/10550/61019/Teis\%20 Doctoral\%20Enrique\%20Madrid\%20\% 281\%29.pdf> (consultado el 9 de enero de 2020).

Valdez, C. y Rufino, O. (2020). Adicción al celular y rasgos de personalidad en estudiantes universitarios de Enfermería (Tesis doctoral). Universidad Nacional de Cajamarca. (Perú). Recuperado de <http://repositorio.upagu. edu.pe/handle/UPAGU/1253> (consultado el 20 de febrero de 2020).

Villa-Moral, M. ${ }^{\text {a }}$ de la y Suárez, C. (2016). Factores de riesgo en el uso problemático de internet y del teléfono móvil en adolescentes españoles. Revista Iberoamericana de Psicología y Salud, 7(2), 69-78. doi: https:// doi.org/10.1016/j.rips.2016.03.001.

Villa-Moral, M. ${ }^{a}$ de la y Fernández-Domínguez, S. (2019). Uso problemático de internet en adolescentes españoles y su relación con autoestima e impulsividad. Avances en Psicología Latinoamericana, 37(1), 103-119. doi: http://dx.doi.org/10.12804/revistas.uro sario.edu.co/apl/a.5029. 
Para los Grados en Derecho o en

Ciencias del Trabajo, Relaciones

Laborales y Recursos Humanos

- Administración y Finanzas

- Asistencia a la Dirección

- Secretariado

\section{Para los Grados en Administración y}

Dirección de Empresas o en Economía

- Administración y Finanzas

- Asistencia a la Dirección

- Comercio Internacional

- Gestión Comercial y Marketing

- Marketing y Publicidad

- Secretariado

- Transporte y Logística

\section{Para el Grado en Marketing}

- Administración y Finanzas

- Comercio Internacional

- Gestión Comercial y Marketing
- Gestión de Alojamientos Turísticos

- Gestión de Ventas y Espacios Comerciales

- Marketing y Publicidad

- Transporte y Logística

Para los Grados en Magisterio de Educación Infantil y de

Educación Primaria

- Animación de Actividades Físicas y Deportivas

- Educación Infantil

- Integración Social

- Animación Sociocultural y Turística

Para el Grado en Empresas y Actividades Turísticas

- Gestión Comercial y Marketing

- Gestión de Ventas y Espacios Comerciales

- Gestión de Alojamientos Turísticos

- Agencias de Viajes y Gestión de Eventos

- Guía, Información y Asistencias Turísticas

\footnotetext{
* De acuerdo a lo establecido en el Real Decreto 1618/2011, de 14 de noviembre, sobre reconocimiento de estudios en el ámbito de la educación superior.
} 


\section{Sedes de examen}

\section{/ Dónde puedes examinarte}

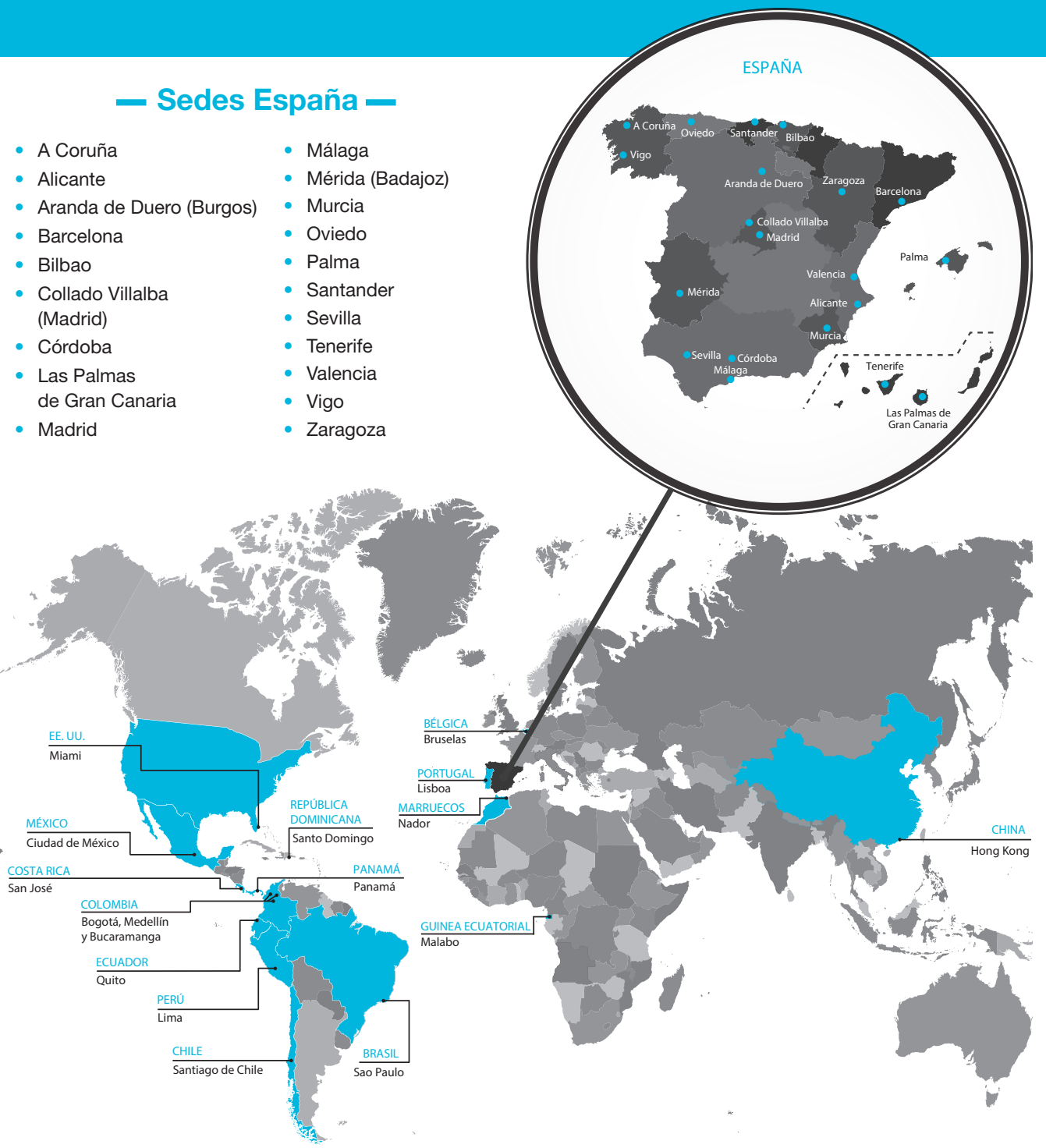

\section{- Sedes extranjero -}

- Bélgica (Bruselas)

- Brasil (Sao Paulo)

- Chile

(Santiago de Chile)

- China

(Hong Kong)
- Colombia (Bogotá, Medellín y

Bucaramanga [sede no permanente])

- Costa Rica (San José)

- Ecuador (Quito)
- Marruecos (Nador [sede no permanente])

- México

(Ciudad de México)

- EE. UU. (Miami)

- Panamá (Panamá)
- Perú (Lima)

- Portugal (Lisboa [sede no permanente])

- República Dominicana (Santo Domingo) 